\title{
SOLUTION OF INTEGRAL EQUATIONS BY PRODUCT INTEGRATION
}

\author{
JON C. HELTON
}

ABSTRACT. Suppose $f, h$ and $G$ are functions with values in a normed complete ring. With suitable restrictions on the sunctions, it is established that

$$
f(x)=h(x)+\int_{a}^{x} f(u) G(u, v)
$$

for $a \leq x \leq b$ only if $\int_{a}^{x} h(u) G(u, v){ }_{v} \Pi^{x}(1+G)$ exists and is $f(x)-h(x)$ for $a \leq x \leq b$, and that

$$
f(x)=h(x)+\int_{a}^{x} G(u, v) f(u)
$$

for $a \leq x \leq b$ only if $\int_{a}^{x} \boldsymbol{\Pi}^{v}(1+\varrho) G(u, v) h(u)$ exists and is $f(x)-h(x)$ for $a \leq x \leq b$, where $\mathcal{S}(s, r)=G(r, s)$.

Lower case letters are used to represent functions from $R$ to $N$, and capital letters are used to represent functions from $R \times R$ to $N$, where $R$ denotes the set of real numbers and $N$ denotes a ring which has a multiplicative identity element represented by 1 and a norm $|\cdot|$ with respect to which $N$ is complete and $|1|=1$. For a subdivision $\left\{x_{i}\right\}_{i=0}^{n}$ of an interval $[a, b]$, we use $G_{i}$ and $f_{i}$ to denote $G\left(x_{i-1}, x_{i}\right)$ and $f\left(x_{i}\right)$, respectively.

The statement that $G \in O B^{\circ}$ on $[a, b]$ means there exist a subdivision $D$ of $[a, b]$ and a number $B$ such that, if $\left\{x_{i}\right\}_{i=0}^{n}$ is a refinement of $D$, then $\sum_{i=1}^{n}\left|G_{i}\right|<B$.

The statement that $\int_{a}^{b} G$ exists means there exists an element $L$ of $N$ such that, if $\epsilon>0$, then there exists a subdivision $D$ of $[a, b]$ such that, if $\left\{x_{i}\right\}_{i=0}^{n}$ is a refinement of $D$, then $\left|L-\sum_{i=1}^{n} G_{i}\right|<\epsilon$. Further, $G \in O A^{\circ}$ on $[a, b]$ only if $\int_{a}^{b} G$ exists and $\int_{a}^{b}\left|G-\int G\right|=0$.

The statement that $a^{b}(1+G)$ exists means there exists an element $L$

Presented to the Society, March 4, 1974; received by the editors January 17, 1974.

AMS (MOS) subject classifications (1970). Primary 45N05; Secondary 26A39, 26A42.

Key words and phrases. Sum integral, product integral, subdivision-refine ment integral, integral equation, interval function, normed complete ring. 
of $N$ such that, if $\epsilon>0$, then there exists a subdivision $D$ of $[a, b]$ such that, if $\left\{x_{i}\right\}_{i=0}^{n}$ is a refinement of $D$, then $\left|L-\Pi_{i=1}^{n}\left(1+G_{i}\right)\right|<\epsilon$. Further, $G \in O M^{\circ}$ on $[a, b]$ only if $\Pi_{x}^{y}(1+G)$ exists for $a \leq x \leq y \leq b$ and $\int_{a}^{b}|1+G-\Pi(1+G)|$ $=0$. The existence of integrals is defined similarly on intervals $[p, q]$, where $q<p$.

The function $h$ is quasi-continuous on $[a, b]$ only if $\lim _{x \rightarrow p^{-}} h(x)$ exists for $a<p \leq b$ and $\lim _{x \rightarrow p^{+}} h(x)$ exists for $a \leq p<b$. Further, $G \in O L^{\circ}$ on $[a, b]$ only if $\lim _{x \rightarrow p^{-}} G(x, p)$ and $\lim _{x, y \rightarrow p}-G(x, y)$ exist for $a<p \leq b$, and $\lim _{x \rightarrow p^{+}} G(p, x)$ and $\lim _{x, y \rightarrow p^{+}} G(x, y)$ exist for $a \leq p<b$. See B. W. Helton [2] and J. S. Mac Nerney [6] for additional background.

The theorems in this paper were suggested by a result of J.S. Mac Nerney [5, Theorem 3.4, p. 361]. However, the ir proofs are constructed by using techniques similar to those employed by B. W. Helton [2, §5, pp. 307-314]. There, the integral equations

$$
f(x)=h(x)+\int_{a}^{x} f(u) G(u, v) \text { and } f(x)=h(x)+\int_{a}^{x} G(u, v) f(u)
$$

were solved with the restriction that $h$ have bounded variation. We now solve these integral equations with the restriction that $h$ be quasi-continuous. The reader is also referred to related results by D. B. Hinton [4, The orems 4.1, 4.2, 4.3, 4.4, pp. 325-327] and C. W. Bitzer [1, Theorems 4.1, 4.2, 5.9, pp. 447-451].

We now state three lemmas that are used in the development of the results in this paper.

Lemma 1. If $G$ is a function from $R \times R$ to $N$ and $G \in O B^{\circ}$ on $[a, b]$, then the following statements are equivalent:

(1) $G \in O A^{\circ}$ on $[a, b]$, and

(2) $G \in O M^{\circ}$ on $[a, b][2$, Theorem 3.4, p. 301].

Lemma 2. If $H$ and $G$ are functions from $R \times R$ to $N, H \in O L^{\circ}$ on $[a, b]$ and $G$ is in $O A^{\circ}$ and $O B^{\circ}$ on $[a, b]$, then $G H$ and $H G$ are in $O A^{\circ}$ and $O M^{\circ}$ on $[a, b][3$, Theorem 2, p. 494].

Lemma 3. If $F$ and $G$ are functions from $R \times R$ to $N$ belonging to $O B^{\circ}$ on $[a, b], F \in O A^{\circ}$ on $[a, b]$ and each of ${ }_{x} \Pi^{y}(1+G)$ and $\int_{x}^{y} F(u, v){ }_{v} \Pi^{y}(1+G)$ exists for $a \leq x<y \leq b$, then

$$
\int_{a}^{b}\left|F(x, y)-\int_{x}^{y} F(u, v) \Pi_{v}^{y}(1+G)\right|
$$

exists and is zero [2, Lemma, p. 307]. 
Theorem 1. If $f$ and $h$ are functions from $R$ to $N, G$ is a function from $R \times R$ to $N, h$ is quasi-continuous on $[a, b]$ and $G \in O B^{\circ}$ on $[a, b]$, then the following statements are equivalent:

(1) $f$ is bounded on $[a, b], G \in O A^{\circ}$ on $[a, b], f(u) G(u, v) \in O A^{\circ}$ on $[a, b]$ and $f(x)=h(x)+\int_{a}^{x} f(u) G(u, v)$ for $a \leq x \leq b$, and

(2) $G \in O M^{\circ}$ on $[a, b]$ and $\int_{a}^{x} h(u) G(u, v){ }_{v} \Pi^{x}(1+G)$ exists and is $f(x)-h(x)$ for $a \leq x \leq b$.

Proof. (1) $\rightarrow(2)$. Since $G$ is in $O A^{\circ}$ and $O B^{\circ}$ on $[a, b]$, it follows from Lemma 1 that $G \in O M^{\circ}$ on $[a, b]$. Suppose $a \leq x \leq b$. If $a=x$, then (2) follows immediately. Therefore, suppose $a<x$. The existence of $I(a, x)$ follows from Lemma 2, where

$$
I(r, s)=\int_{r}^{s} h(u) G(u, v) \prod_{v}^{s}(1+G)
$$

for $a \leq r \leq s \leq b$. We now show that $I(a, x)$ is equal to $f(x)-b(x)$. Let $\epsilon>0$.

There exists a subdivision $D_{1}$ of $[a, x]$ such that, if $\left\{x_{i}\right\}_{i=0}^{n}$ is a refinement of $D_{1}$, then

$$
\left|I(a, x)-\sum_{i=1}^{n} h_{i-1} G_{i} \prod_{i} \prod^{x}(1+G)\right|<\epsilon / 3 .
$$

There exist a subdivision $D_{2}$ of $[a, x]$ and a number $B$ such that, if $\left\{x_{i}\right\}_{i=0}^{n}$ is a refinement of $D_{2}$, then

(1) $\prod_{i=1}^{n}\left(1+\left|G_{i}\right|\right)<B$, and

(2) $\sum_{i=1}^{n}\left|h_{i-1} G_{i}\right|<B$.

Since $G$ is in $O M^{\circ}$ and $O B^{\circ}$ on $[a, x]$, there exists a subdivision $D_{3}$ of $[a, x]$ such that, if $\left\{x_{i}\right\}_{i=0}^{n}$ is a refinement of $D_{3}$ and $1 \leq i \leq n$, then

$$
\left|\prod_{i} \prod^{x}(1+G)-\prod_{k=i+1}^{n}\left(1+G_{k}\right)\right|<\epsilon(3 B)^{-1} .
$$

Since $f(u) G(u, v) \in O A^{\circ}$ on $[a, x]$, there exists a subdivision $D_{4}$ of $\left[a,^{\prime} x\right]$ such that, if $\left\{x_{i}\right\}_{i=0}^{n}$ is a refinement of $D_{4}$, then

$$
\sum_{i=1}^{n}\left|f_{i-1} G_{i}-\int_{x_{i-1}}^{x_{i}} f(u) G(u, v)\right|<\epsilon(3 B)^{-1} .
$$

Let $D$ denote the subdivision $\bigcup_{i=1}^{4} D_{i}$ of $[a, x]$. Suppose $\left\{x_{i}\right\}_{i=0}^{n}$ is a refinement of $D$. F or $1 \leq i \leq n$, we have that 


$$
f_{i}-f_{i-1}=h_{i}-h_{i-1}+f_{i-1} G_{i}+c_{i} \text {, }
$$

where

$$
c_{i}=\int_{x_{i-1}}^{x_{i}} f(u) G(u, v)-f_{i-1} G_{i}
$$

Thus,

$$
f_{i}=h_{i}-h_{i-1}+f_{i-1}\left(1+G_{i}\right)+c_{i}
$$

Now, by using iteration for $i=1,2, \cdots, n$, we have that

$$
f_{n}=h_{n}+\sum_{i=1}^{n} h_{i-1} G_{i} \prod_{k=i+1}^{n}\left(1+G_{k}\right)+\sum_{i=1}^{n} c_{i} \prod_{k=i+1}^{n}\left(1+G_{k}\right) \text {. }
$$

Hence,

$$
\begin{aligned}
& |I(a, x)-\{f(x)-h(x)\}| \\
& =\mid h(x)+I(a, x)-h_{n} \\
& -\sum_{i=1}^{n} h_{i-1} G_{i} \prod_{k=i+1}^{n}\left(1+G_{k}\right)-\sum_{i=1}^{n} c_{i} \prod_{k=i+1}^{n}\left(1+G_{k}\right) \\
& \leq\left|\sum_{i=1}^{n} h_{i-1} G_{i_{x}} \prod^{x}(1+G)-\sum_{i=1}^{n} h_{i-1} G_{i} \prod_{k=i+1}^{n}\left(1+G_{k}\right)\right| \\
& +\left|I(a, x)-\sum_{i=1}^{n} h_{i-1} G_{i_{x}} \prod^{x}(1+G)\right|+\sum_{i=1}^{n}\left|-c_{i}\right|\left|\prod_{k=i+1}^{n}\left(1+G_{k}\right)\right| \\
& <\left.\sum_{i=1}^{n}\left|h_{i-1} G_{i}\right|\right|_{x_{i}} \prod^{x}(1+G)-\prod_{k=i+1}^{n}\left(1+G_{k}\right) \mid+\epsilon / 3+B\left[\epsilon(3 B)^{-1}\right] \\
& <B\left[\epsilon(3 B)^{-1}\right]+2 \epsilon / 3=\epsilon .
\end{aligned}
$$

Therefore, (1) implies (2).

Proof. (2) $\rightarrow(1)$. Since $h$ is bounded on $[a, b]$ and $G \in O B^{\circ}$ on $[a, b]$, it follows that $f$ is bounded on $[a, b]$. Since $G$ is in $O M^{\circ}$ and $O B^{\circ}$ on $[a, b]$, it follows from Lemma 1 that $G \in O \dot{A}^{\circ}$ on $[a, b]$. Further, it follows by employing Lemma 2 that $f(u) G(u, v) \in O A^{\circ}$ on $[a, b]$. Suppose $a \leq x \leq b$. If $a=x$, then (2) follows immediately. Therefore, suppose $a<x$. Let $\epsilon>0$.

There exists a subdivision $D_{1}$ of $[a, x]$ such that, if $\left\{x_{i}\right\}_{i=0}^{n}$ is a refinement of $D_{1}$, then

$$
\left|\int_{a}^{x} f(u) G(u, v)-\sum_{i=1}^{n} f_{i-1} G_{i}\right|<\frac{\epsilon}{3}
$$


There exists a number $B$ such that, if $a \leq t \leq x$, then $|I(a, t)|<B$, where $I(a, t)$ is defined as in the first part of the proof. Now, since $G \in O M^{\circ}$ on $[a, x]$, there exists a subdivision $D_{2}$ of $[a, x]$ such that, if $\left\{x_{i}\right\}_{i=0}^{n}$ is a refinement of $D_{2}$, then

$$
\sum_{i=1}^{n}\left|1+G_{i}-x_{i-1} \prod^{x}(1+G)\right|<\epsilon(3 B)^{-1} .
$$

It follows by applying Lemma 3 that there exists a subdivision $D_{3}$ of $[a, x]$ such that, if $\left\{x_{i}\right\}_{i=0}^{n}$ is a refinement of $D_{3}$, then

$$
\sum_{i=1}^{n}\left|h_{i-1} G_{i}-I\left(x_{i-1}, x_{i}\right)\right|<\frac{\epsilon}{3} .
$$

Let $D$ denote the subdivision $\bigcup_{i=1}^{3} D_{i}$ of $[a, x]$. Suppose $\left\{x_{i}\right\}_{i=0}^{n}$ is a refinement of $D$. Thus,

$$
\begin{aligned}
\mid h(x) & +\int_{a}^{x} f(u) G(u, v)-f(x) \mid \\
\leq & \left|h(x)+\sum_{i=1}^{n} f_{i-1} G_{i}-h(x)-I(a, x)\right|+\left|\int_{a}^{x} f(u) G(u, v)-\sum_{i=1}^{n} f_{i-1} G_{i}\right| \\
& <\left|\sum_{i=1}^{n}\left\{h_{i-1}+I\left(a, x_{i-1}\right)\right\} G_{i}-I(a, x)\right|+\epsilon / 3 \\
= & \left|\sum_{i=1}^{n}\left\{h_{i-1} G_{i}+\left[I\left(a, x_{i-1}\right)\right]\left[1+G_{i}\right]-I\left(a, x_{i-1}\right)\right\}-I(a, x)\right|+\epsilon / 3 \\
\leq & \left|\sum_{i=1}^{n}\left\{h_{i-1} G_{i}+\left[I\left(a, x_{i-1}\right)\right]\left[{ }_{i-1} \prod^{x}(1+G)\right]-I\left(a, x_{i-1}\right)\right\}-I(a, x)\right| \\
& +\sum_{i=1}^{n}\left|I\left(a, x_{i-1}\right)\right|\left|1+G_{i}-{ }_{x} \prod^{x}(1+G)\right|+\epsilon / 3 \\
< & \left|\sum_{i=1}^{n}\left\{I\left(a, x_{i}\right)-I\left(a, x_{i-1}\right)\right\}-I(a, x)\right| \\
& +\sum_{i=1}^{n}\left|h_{i-1} G_{i}-I\left(x_{i-1}, x_{i}\right)\right|+B\left[\epsilon(3 B)^{-1}\right]+\epsilon / 3 \\
< & +\epsilon / 3+2 \epsilon / 3=\epsilon .
\end{aligned}
$$

Therefore, (2) implies (1). 
Theorem 2. If $f$ and $h$ are functions from $R$ to $N, G$ is a function from $R \times R$ to $N, \mathcal{G}(y, x)=G(x, y)$ for $a \leq x<y \leq b, h$ is quasi-continuous on $[a, b]$ and $G \in O B^{\circ}$ on $[a, b]$, then the following statements are equivalent:

(1) $f$ is bounded on $[a, b], G \in O A^{\circ}$ on $[a, b], G(u, v) f(u) \in O A^{\circ}$ on $[a, b]$ and $f(x)=h(x)+\int_{a}^{x} G(u, v) f(u)$ for $a \leq x \leq b$, and

(2) $\Theta \in O M^{\circ}$ on $[b, a]$ and $\int_{a}^{x} \Pi^{v}(1+\mathcal{G}) G(u, v) h(u)$ exists and is $f(x)-h(x)$ for $a \leq x \leq b$.

The proof of Theorem 2 is similar to the proof of Theorem 1, and therefore, we omit it.

\section{REFERENCES} 434-451.

1. C. W. Bitzer, Stieltjes-Volterra integral equations, Illinois J. Math. 14 (1970),

2. B. W. Helton, Integral equations and product integrals, Pacific J. Math. 16 (1966), 297-322. MR $32 \# 6167$.

3. - - A product integral representation for a Gronwall inequality, Proc. Amer. Math. Soc. 23 (1969), 493-500. MR 40 \#1562.

4. D. B. Hinton, A Stieltjes-Volterra integral equation theory, Canad. J. Math. 18 (1966), 314-331. MR 32 \#6169.

5. J. S. Mac Nerney, Stieltjes integrals in linear spaces, Ann. of Math. (2) 61 (1955), 354-367. MR 16, 716.

6. - Integral equations and semigroups, Illinois J. Math. 7 (1963), 148173. MR $26 \# 1726$.

DEPARTMENT OF MATHEMATICS, ARIZONA STATE UNIVERSITY, TEMPE, ARIZONA 85281 